\title{
Thermal diffusivity measurement using the photopyroelectric effect
}

\author{
P. K. John, L. C. M. Miranda, ${ }^{*}$ and A. C. Rastogi ${ }^{\dagger}$ \\ Centre for Chemical Physics, University of Western Ontario, London, Ontario, Canada N6A $3 K 7$
}

(Received 22 May 1986)

\begin{abstract}
A novel and simple method using the photopyroelectric effect is described for the measurement of the thermal diffusivity of solids. The method is used in the case of metals, semiconductors, and insulators, covering several orders of magnitude in thermal diffusivity. It has advantages over conventional thermal wave spectroscopic methods.
\end{abstract}

In the last few years a great deal of effort has been devoted to the development of thermal wave spectroscopies, such as photoacoustic spectroscopy (PAS), ${ }^{1,2}$ photothermal radiometry, ${ }^{3,4}$ and photothermal deflection spectroscopy (PDS) ${ }^{5-7}$ The common basic principle of these thermal spectroscopies consists of measuring the temperature fluctuation in a sample as a result of the nonradiative deexcitation processes that take place following the absorption of radiation. Apart from providing us with optical absorption spectra these techniques exhibit the unique feature of allowing us to measure the depth profile ${ }^{8-11}$ (microscopy) of samples as well as their thermal properties. ${ }^{12-16}$ More recently, Coufal, ${ }^{17}$ Mendelis, ${ }^{18}$ and Ghizoni and Miranda ${ }^{19}$ have opened the way to a new technique using pyroelectric thin films to detect optical absorption and nonradiative energy conversion processes in solids. This new technique consists of using a thin pyroelectric [polyvinylidene difluoride $\left(\mathrm{PVF}_{2}\right)$ ] film in intimate contact with a solid sample on which a monochromatic light beam whose intensity is sinusoidally modulated at a frequency $\omega$ is incident. Following the absorption of the incident light, the nonradiative deexcitation processes within the solid cause the sample temperature to fluctuate and, through heat diffusion to the surrounding pyroelectric film, the temperature of the sample-pyroelectric-film interface fluctuates, thereby creating a temperature gradient in the pyroelectric film. As a result of this temperature gradient, the pyroelectric film changes its polarization, thus producing a net dipole moment which results in a pyroelectric voltage $V$ given by ${ }^{20}$

$$
V=\frac{p l_{d}}{\epsilon} \Delta T
$$

where $p$ is the pyroelectric coefficient of the film, $l_{d}$ is the film thickness, $\epsilon$ is the film dielectric constant, and $\Delta T$ is the spatially averaged temperature fluctuation in the pyroelectric film, namely,

$$
\Delta T=\frac{1}{l_{d}} \int T_{d}(x, t) d x .
$$

In the experiments by Coufal ${ }^{17}$ the pyroelectric character of 9- $\mu \mathrm{m}$ thin $\mathrm{PVF}_{2}$ films was used to obtain the absorption characteristics of $\mathrm{Nd}_{2} \mathrm{O}_{3}$-doped polymethyl methaacrylate films. In the work by Mandelis the potentiality of this new photopyroelectric technique was demonstrated by using this technique for obtaining the optical spectrum of $\mathrm{Ho}_{2} \mathrm{O}_{3}$ hydrated powders as well as to monitor the kinetics of the chemical reduction of $\mathrm{CuO}$ into metallic copper by $\mathrm{HCl}$. Ghizoni and Miranda ${ }^{19}$ have used the method to measure thermal diffusivity of silicon crystal using a lateral heating source.

In this paper we describe the application and potential of the photopyroelectric effect as a new, simple, and sensitive method for measuring the thermal properties of solids, covering the range from conductors, through semiconductors, to insulators. This approach is schematically shown in Fig. 1. A monochromatic light beam whose intensity is modulated at a frequency $\omega=2 \pi f$ is incident on a solid sample of thickness $l_{s}$. The sample is in intimate contact with the thin $\mathrm{PVF}_{2}$ film of thickness $l_{d}$, by means of a thin coupling fluid layer of thickness $l_{c}$, and the sampletransducer system is supported by an optically transparent backing material of thickness $l_{b}$, large compared with $l_{s}$, $l_{c}$, and $l_{d}$. The temperature fluctuation in material $i$ ( $i=g, c, d, b)$ of our system resulting from the periodic optical heating of the sample denoted by $T_{i}(x, t)$ can be obtained by solving the coupled set of thermal diffusion equations,

$$
\frac{\partial^{2} T_{i}}{\partial x^{2}}-\frac{1}{\alpha_{i}} \frac{\partial T_{i}}{\partial T}=\left\{\begin{array}{l}
S(x, t), i=s, \\
0, i=g, c, d, b,
\end{array}\right.
$$

where the heat density $S(x, t)$ produced in the sample by the absorption of light is given by

$$
S(x, t)=\frac{\beta^{\prime} I_{0}}{2} \delta\left(x-l_{s}\right) e^{j \omega t} .
$$

In Eqs. (3) and (4), $k_{i}$ is the thermal conductivity of material $i ; \alpha_{i}=k_{i} / \rho_{i} c_{i}$ is the thermal diffusivity of material $i$, where $\rho_{i}$ is the density of material $i ; c_{i}$ is the specific heat

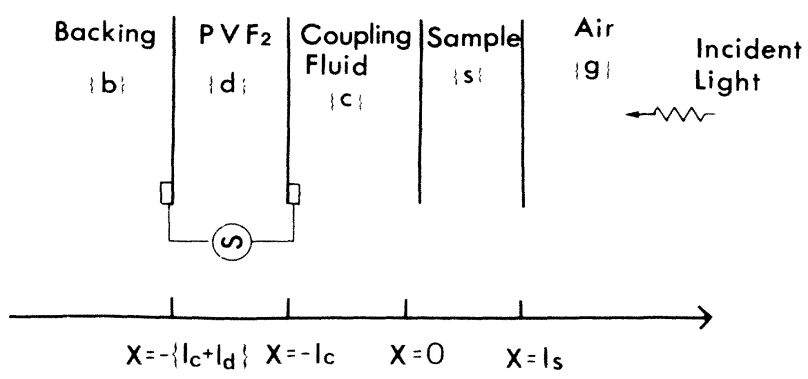

FIG. 1. Configuration of a photopyroelectric system. 
of material $i, I_{0}$ is the intensity of the incident light, and $\beta^{\prime}$ is the surface absorption coefficient of the sample. The quantity of interest is $T_{d}(x, t)$ and the actual temperature fluctuation in the pyroelectric film as a function of position and time is given by the real part of $T_{d}(x, t)$. Solving Eq. (3) together with the boundary conditions of heat-flux and temperature continuity at the interfaces one finds the periodic temperature fluctuation in the pyroelectric film. Knowing $T_{d}(x, t)$ and performing the spatial average indicated in Eq. (2), one gets

$$
\Delta T=\left(\frac{2 c \beta^{\prime} I_{0}}{k_{s} \sigma_{s} l_{d} \sigma_{d}}\right)\left(\frac{\left(1-e^{-l_{d} \sigma_{d}}\right)-\gamma\left(1-e^{l_{d} \sigma_{d}}\right)}{\left[(1+c) e^{l_{s} \sigma_{s}}-(1-c) e^{-l_{s} \sigma_{s}}\right] \xi_{1} e^{l_{c} \sigma_{c}}+\left[(1-c) e^{l_{s} \sigma_{s}}-(1+c) e^{-l_{s} \sigma_{s}}\right] \xi_{2} e^{-l_{c} \sigma_{c}}}\right),
$$

where $\sigma_{i}=(1+j) a_{i}, i=b, c, d, g, s, a_{i}=\left(\omega / 2 \alpha_{i}\right)^{1 / 2}=\mu_{i}^{-1}$ is the thermal diffusion coefficient, $\mu_{i}$ is the thermal diffusion length, $\xi_{1}=c(1+\gamma)+d(1-\gamma)$ and $\xi_{2}=c(1+\gamma)$ $-d(1-\gamma)$. Here $b=k_{b} a_{b} / k_{s} a_{s}, c=k_{c} a_{c} / k_{s} a_{s}, d=k_{d} a_{d} /$ $k_{s} a_{s}$, and $\gamma=[(d-b) /(d+b)] \exp \left(-2 l_{d} \sigma_{d}\right)$.

The above expression for the average temperature fluctuation in the pyroelectric film is quite complicated to interpret in the general case. It, however, reduces to simpler expressions in some limiting cases depending on the thermal thickness (i.e., the product $l_{s} \sigma_{s}$ ) of our sample. For the purpose of measuring the thermal diffusivity, the case of interest is that of thermally thick sample, namely, when $l_{s} \sigma_{s} \gg 1$. We note that both the pyroelectric film and the coupling fluid may be considered thermally thin. Thus, setting in Eq. (5) $\exp \left(-l_{s} \sigma_{s}\right)=0, \exp \left( \pm l_{c} \sigma_{c}\right) \simeq 1$ $\pm l_{c} \sigma_{c}$, and $\exp \left( \pm l_{d} \sigma_{d}\right)=1 \pm l_{d} \sigma_{d}$ the temperature fluctuation in the pyroelectric film should then decay exponentially for $l_{s} \sigma_{s} \gg 1$, i.e.,

$$
\Delta T=e^{-\sqrt{f / f_{s}}}, \text { where } f_{s}=\alpha_{s} / \pi l_{s}^{2} .
$$

This exponential decay of the signal with the modulation frequency depends only on the thermal diffusion time in the sample $f_{s}^{-1}$ and, consequently, allows us to measure the thermal diffusivity, provided the sample thickness is known.

To demonstrate the versatility of the above method for measuring thermal diffusivity we have carried out the measurements on copper, on silicon crystal wafer, and on glass (microscope cover plate). The experimental setup is shown in Fig. 2. The beam from a $1-\mathrm{mW} \mathrm{He}-\mathrm{Ne}$ laser modulated by a variable frequency chopper is focused on the sample which is in thermal contact with a $9-\mu \mathrm{m}$-thick $\mathrm{PVF}_{2}$ film (Pennwalt Corp.) supported by a Plexiglass backing. To optimize the sample-PVF $\mathrm{PV}_{2}$ thermal contact a thin $(50-100-\mu \mathrm{m}$ thick) layer of thermally conducting grease (thermal compound No. 120-8, Wakefield, England) was used as the coupling fluid. The output from the $\mathrm{PVF}_{2}$ detector after preamplification was fed to a lock-in amplifier. In Figs. 3 and 4 we show the variation of the pyroelectric signals as a function of $\sqrt{f}$ for the $\mathrm{Si}$ $\left(l_{s}=0.37-\mathrm{mm}\right)$ and $\mathrm{Cu}\left(l_{s}=0.50-\mathrm{mm}\right)$ samples, respectively. Figure 5 shows the corresponding changes in signal for a thin microscope cover glass plate $\left(l_{s}=60 \mu \mathrm{m}\right)$. The front surface of the glass plate was coated with a thin carbon-black layer to ensure the optically thick condition for the $\mathrm{He}-\mathrm{Ne}$ laser light. The semilog plots of Figs. 3 and 4 clearly show two distinct straight-line regions as a function of $\sqrt{f}$. These features can be understood from Eq. (5) as follows. For the materials used in our work in the frequency range where the samples are thermally thick one has $d / b=0.97, \gamma \cong 0, \xi_{1}=c+d$, and $\xi_{2}=c-d$. Making these substitutions in Eq. (5), and neglecting $\exp \left(-l_{d} \sigma_{d}\right)$ as compared to one, we get for a thermally thick sample,

$$
\Delta T=\frac{c \beta^{\prime} I_{0}}{k_{s} \sigma_{s} l_{d} \sigma_{d}} \frac{e^{-l_{s} \sigma_{s}}}{\left[c \cosh \left(l_{c} \sigma_{c}\right)+d \sinh \left(l_{c} \sigma_{c}\right)\right]} .
$$

Equation (7) shows that as long as the coupling fluid is not thermally thick (i.e., as long as $l_{c} \sigma_{c}<1$ ) the exponential decay of the pyroelectric signal, as a function of $\sqrt{f}$, is entirely due to the sample and varies as $\exp \left(-\sqrt{f / f_{s}}\right)$

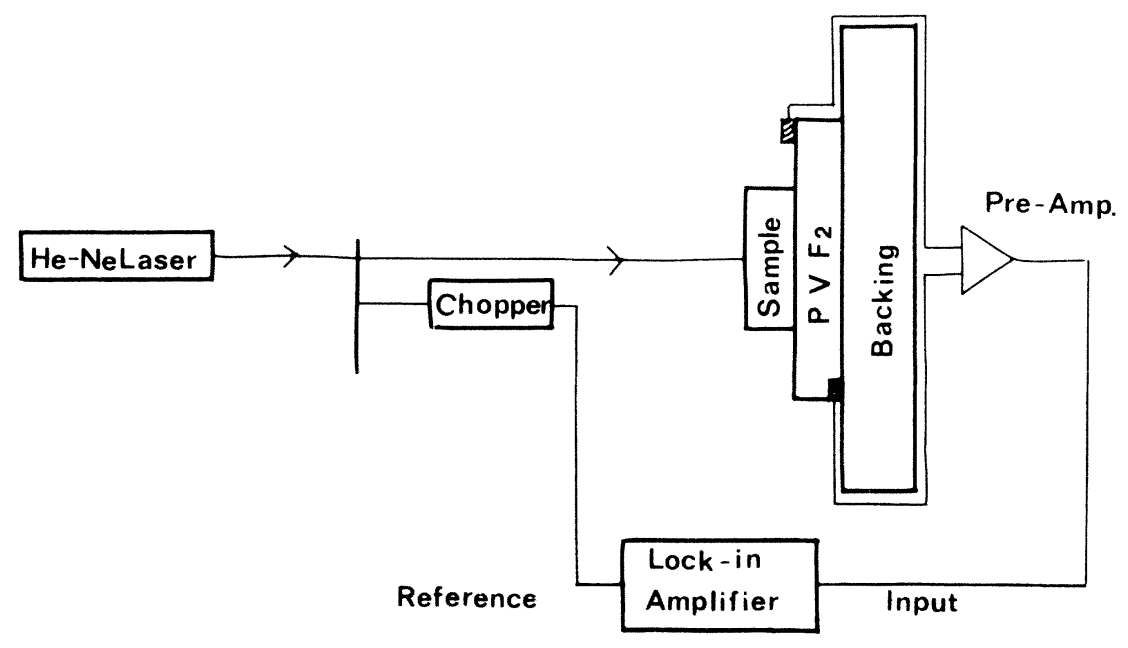

FIG. 2. Schematic of the experimental setup used for measuring the thermal diffusivity. 


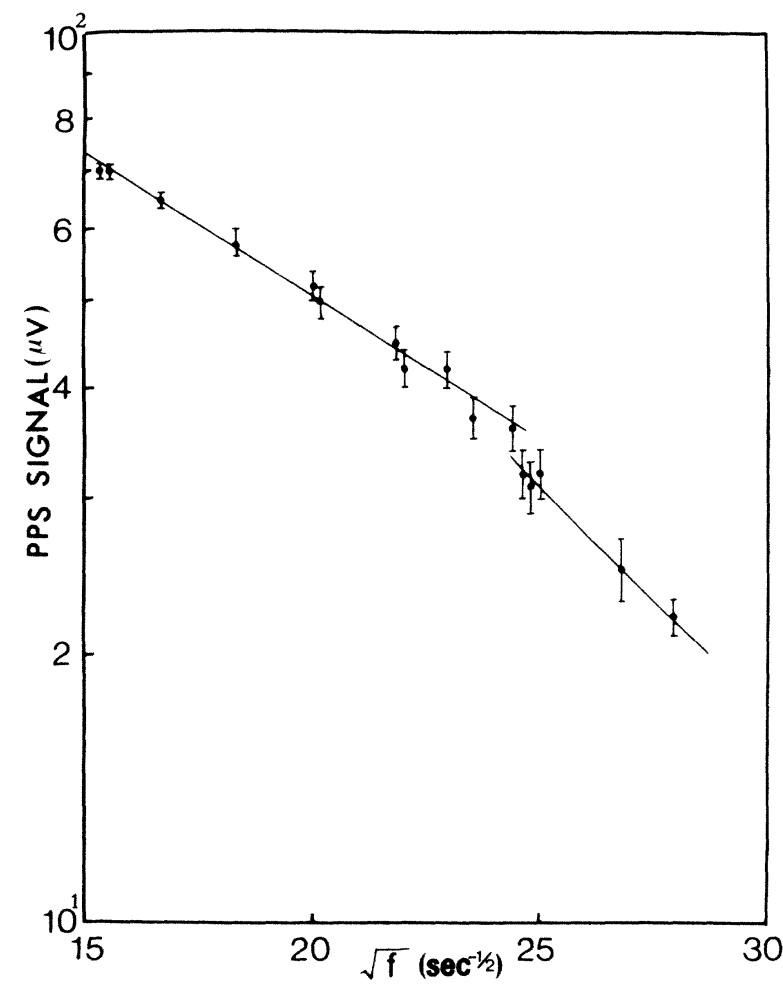

FIG. 3. Semilog plot of the phyotpyroelectric signal (PPS) of a Silicon wafer, $370 \mu \mathrm{m}$ thick, as a function of the square root of the modulation frequency. The solid lines represent the leastsquares linear fitting of the experimental data.

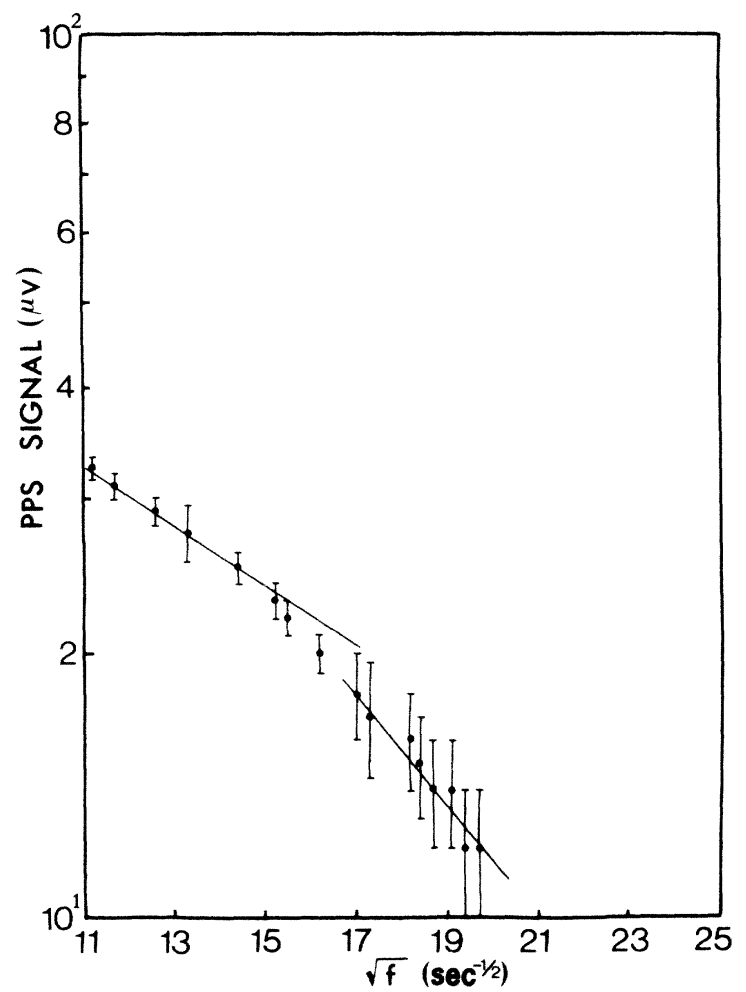

FIG. 4. Semilog plot of the photopyroelectric signal of a $\mathrm{Cu}$ plate, $500 \mu \mathrm{m}$ thick, as a function of the square root of the modulation frequency. The solid lines represent the leastsquares linear fitting of the experimental data.

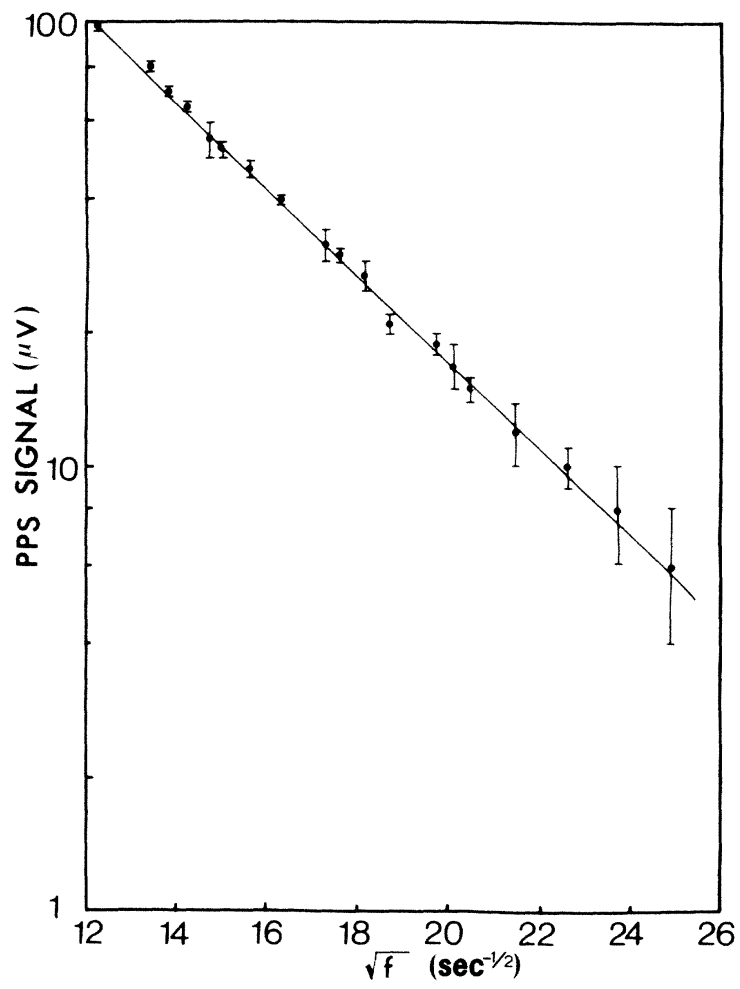

FIG. 5. Semilog plot of the photoelectric signal of a cover glass plate, $60 \mu \mathrm{m}$ thick, as a function of the square root of the modulation frequency. The solid line represents the leastsquares linear fitting of the experimental data.

where $f_{s}=\alpha_{s} / \pi l_{s}^{2}$. As one increases the modulation frequency one eventually reaches a situation where the coupling fluid becomes thermally thick (i.e., $l_{c} \sigma_{c}>1$ ). At this point the exponential decay is due to both the sample and the coupling fluid and the pyroelectric signal varies as $\exp \left[-\left(f_{s}^{-1 / 2}+f_{c}^{-1 / 2}\right) \sqrt{f}\right]$, where $f_{c}=\alpha_{c} / \pi l_{c}^{2}$. This means that by performing a least-squares fitting of the straight-line regions of the logarithm of the pyroelectric signal as a function of $\sqrt{f}$, one is able to calculate the thermal diffusivity of both the sample and the coupling fluid. In the case of $\mathrm{Si}$ (Fig. 3), the fitting in the region between 200 and $600 \mathrm{~Hz}$ yielded $f_{s}=182.6 \mathrm{~Hz}$ from which $\alpha_{s}=\pi l_{s}^{2} f_{s}$ can be calculated, which gives $\alpha_{s}=0.8 \mathrm{~cm}^{2} / \mathrm{s}$. This value of $\alpha_{s}$ in in very good agreement with the published values of $\mathrm{Si}$ as given in Table I. From the fitting in the region between 600 and $800 \mathrm{~Hz}$ we obtained $f_{c}=567$ $\mathrm{Hz}$. Using the measured value of the thermal compound thickness, $l_{c}=25 \mu \mathrm{m}$, the value of $\alpha_{c}=\pi l_{c}^{2} f_{c}$ was then calculated to be $\alpha_{c}=0.012 \mathrm{~cm}^{2} / \mathrm{s}$. Similarly in the case of $\mathrm{Cu}$ (Fig. 4), the least-squares fittings in the two linear regions yielded $f_{s}=151.6 \mathrm{~Hz}$ and $f_{c}=244 \mathrm{~Hz}$. Using the thickness of the thermal compound layer in this case $l_{c} \sim 50 \mu \mathrm{m}$ and of the copper foil $l_{s}=500 \mu \mathrm{m}$, the calculated values of $\alpha_{s}$ and $\alpha_{c}$ were $\alpha_{s}=1.2 \mathrm{~cm}^{2} / \mathrm{s}$ and $\alpha_{c}=0.019 \mathrm{~cm}^{2} / \mathrm{s}$. Similar to the measurements of $\mathrm{Si}$, the agreement between our measured value for the thermal diffusivity of $\mathrm{Cu}$ and the value of $\alpha_{s}=1.15 \mathrm{~cm}^{2} / \mathrm{s}$ quoted in the literature (see Table I) is very good. As for the values of $\alpha_{c}$ of the thermal compound obtained from the two experiments, the agreement 
TABLE I. Thermal diffusivity $(\alpha)$ for the materials used in our experiment.

\begin{tabular}{llll}
\hline \hline \multicolumn{1}{c}{ Material } & \multicolumn{1}{c}{$\mathrm{Si}$} & \multicolumn{1}{c}{$\mathrm{Cu}$} & Glass \\
\hline $\begin{array}{l}\text { Measured } \\
\text { value }\left(\mathrm{cm}^{2} / \mathrm{s}\right)\end{array}$ & 0.9 & 1.15 & 0.002 \\
$\begin{array}{l}\text { Standard } \\
\text { value }\left(\mathrm{cm}^{2} / \mathrm{s}\right)\end{array}$ & 0.9 (Ref. 22) & 1.2 (Ref. 23) & 0.004 (Ref. 24) \\
\hline \hline
\end{tabular}

is within the error limits ${ }^{21}$ at high frequencies. In the case of the glass plate the semilog plot of the pyroelectric signal as a function of $\sqrt{f}$ exhibited just one straight line as shown in Fig. 5. The reason for this is that for glasses the thermal diffusivity $\left(\alpha \sim 10^{-3} \mathrm{~cm}^{2} / \mathrm{s}\right)$ is much smaller than that of metals and semiconductors. This means that for the case of glasses, on increasing the modulation frequency, the pyroelectric signal would die out before the coupling fluid becomes thermally thick. The least-squares linear fitting of the logarithm of the pyroelectric signal as a function $\sqrt{f}$ yielded $f_{s}=17.3 \mathrm{~Hz}$ from which we get $\alpha_{s}=0.002 \mathrm{~cm}^{2} / \mathrm{s}$.

In conclusion, we have demonstrated in this paper the potential use of the photopyroelectric effect for measuring the thermal diffusivity of solids over a very wide range. The specific examples we have considered varied from the high thermal diffusivity (metals) to the low thermal diffusivity (glasses) materials covering a range of more than 4 orders of magnitude in thermal diffusivity. The extreme simplicity, sensitivity, as well as its adaptability to practi- cal restrictions imposed by experimental systems requirements suggest that photopyroelectric method is a potentially powerful technique, especially when in situ nondestructive testing of samples with minimal preparation is required.

Two of us (L.C.M.M. and A.C.R.) gratefully acknowledge the hospitality extended to us by the Centre for Interdisciplinary Studies in Chemical Physics. One of us (L.C.M.M.) also acknowledges the financial support from the Centro Technico Aeroespacial, the National Sciences and Engineering Research Council of Canada, the Brazilian National Research Council, and the State of São Paulo Government through its agency Fundaçao de Ampero e Pesquisa do Estado de São Paulo. The many useful discussions on photopyroelectric spectroscopy with Professor A. Mandelis of the University of Toronto, who kindly supplied the $\mathrm{PVF}_{2}$ sample used in this work, are gratefully acknowledged.
* On leave of absence from the Instituto de Estudos Avandados, Centro Technico Aerospacial, 12200, S.J. Campos, São Paulo, Brazil.

ton leave of absence from the National Physical Laboratory, Hillside Road, New Delhi 110012, India.

${ }^{1}$ A. Rosencwaig and A. Gersho, J. Appl. Phys. 47, 64 (1976).

${ }^{2}$ A. C. Tam and C. K. N. Patel, Rev. Mod. Phys. 53, 517 (1981).

${ }^{3}$ P. E. Nordal and S. O. Kanstad, Phys. Scr. 20, 659 (1979).

${ }^{4}$ R. Santos and L. C. M. Miranda, J. Appl. Phys. 52, 4194 (1981).

${ }^{5}$ A. C. Boccara, D. Fournier, and J. Badoz, Appl. Phys. Lett. 36, 130 (1980)

${ }^{6}$ J. C. Murphy and L. C. Aamodt, J. Appl. Phys. 51, 4580 (1980).

${ }^{7}$ W. D. Jackson, N. M. Amer, A. C. Boccara, and D. Fournier, Appl. Opt. 20, 1333 (1981).

${ }^{8}$ Y. H. Wong, R. L. Thomas, and G. F. Hawkins, Appl. Phys. Lett. 32, 538 (1978).

${ }^{9}$ A. Rosencwaig, J. Photoacoust. 1, 75 (1982).

${ }^{10}$ C. L. Cesar, H. Vargas, J. Pelzl, and L. C. M. Miranda, J. Appl. Phys. 55, 3460 (1984).

${ }^{11}$ R. S. Quimby, Appl. Phys. Lett. 45, 1037 (1984).

${ }^{12}$ M. J. Adams and G. F. Kirkbright, Analyst (London) 102, 678 (1977).
${ }^{13}$ M. A. A. Siqueira, C. C. Ghizoni, J. I. Vargas, E. Menezes, H. Vargas, and L. C. M. Miranda, J. Appl. Phys. 51, 1403 (1980).

${ }^{14}$ P. Charpentier, F. Lepoutre, and L. Bertrand, J. Appl. Phys. 53, 608 (1982).

${ }^{15}$ C. L. Cesar, H. Vargas, J. Mendes Filho, and L. C. M. Miranda, Appl. Phys. Lett. 43, 555 (1983).

${ }^{16}$ A. Lachaine and P. Poulet, Appl. Phys. Lett. 45, 953 (1984).

${ }^{17}$ H. Coufal, Appl. Phys. Lett. 44, 59 (1984).

${ }^{18}$ A. Mandelis, Chem. Phys. Lett. 108, 388 (1984).

${ }^{19}$ C. C. Ghiszoni and L. C. M. Miranda, Phys. Rev. B 32, 8392 (1985).

${ }^{20}$ Kynar $^{T M}$ Piezo Film Technical Manual (Pennwalt Corp., King of Prussia, PA, 1983), p. 17.

${ }^{21}$ D. Grzegorczyk and G. Feineman, in Handbook of Platics in Electronics (Reston, Reston, VA, 1974), pp. 16-31.

${ }^{22}$ S. M. Sze, Physics of Semiconductor Devices (Wiley, New York, 1981).

${ }^{23}$ American Institute of Physics Handbook (McGraw-Hill, New York, 1982).

${ }^{24}$ Handbook of Chemistry and Physics (Chemical Rubber Co., Cleveland, $\mathrm{OH}, 1964)$. 